\title{
Estimation of Speciation Data for Hydrocarbons using Data Science
}

\author{
Author, co-author (Do NOT enter this information. It will be pulled from participant tab in \\ MyTechZone) \\ Affiliation (Do NOT enter this information. It will be pulled from participant tab in MyTechZone)
}

\begin{abstract}
Strict regulations on air pollution motivates clean combustion research for fossil fuels. To numerically mimic real gasoline fuel reactivity, surrogates are proposed to facilitate advanced engine design and predict emissions by chemical kinetic modelling. However, chemical kinetic models could not accurately predict non-regular emissions, e.g. aldehydes, ketones and unsaturated hydrocarbons, which are important air pollutants. In this work, we propose to use machine-learning algorithms to achieve better predictions. Combustion chemistry of fuels constituting of 10 neat fuels, 6 primary reference fuels (PRF) and 6 FGX surrogates were tested in a jet stirred reactor. Experimental data were collected in the same setup to maintain data uniformity and consistency under following conditions: residence time at 1.0 second, fuel concentration at $0.25 \%$, equivalence ratio at 1.0 , and temperature range from 750 to $1100 \mathrm{~K}$. Measured species profiles of methane, ethylene, propylene, hydrogen, carbon monoxide and carbon dioxide are used for machine-learning model development. The model considers both chemical effects and physical conditions. Chemical effects are described as different functional groups, viz. primary, secondary, tertiary, and quaternary carbons in molecular structures, and physical conditions as temperature. Both the Machine-learning models used in this study showed a good prediction accuracy with a test set regression score of 97.75 for support vector regression and 91.07 for random forest regression. This finding shows the great potential of machine learning application on combustion chemistry. By expanding the experimental database, machine-learning models can be further applied to many other hydrocarbons in future work.
\end{abstract}

\section{Introduction}

Gasoline fuels play a key role in current century. The main energy source for light-duty vehicles is currently gasoline, and will remain so in the near future. Twenty percent of world's total energy is used in transportation sector, and $40 \%$ of transportation energy is used for light duty vehicles [1]. While this satisfy our transportation needs, large amount of pollutants are released, causing severe air pollution. Advancements in combustion research are therefore required for reduction in emissions and to comply with strict regulations.

Surrogate fuels are proposed to numerically mimic complex gasoline fuels. Surrogates consist of a few hydrocarbon molecules that are usually key components of gasoline fuels. Proposed surrogates should mimic real gasoline fuel properties and behavior. They should have similar key properties with real fuels, e.g. density, average molecular weight, hydrogen to carbon ratio, research octane number, motor octane number and octane density [2]. Besides fuel properties,

Page 1 of 9 surrogates should also have similar reactivity with real gasoline fuels. A successful surrogate should be able to represent real fuels on ignition delay time [3-5], flame speed [6] and speciation distributions in ideal reactors, e.g. jet stirred reactors (JSRs) [7-9] and laminar premixed flames [10].

Among all the proposed surrogates for gasoline fuels, the simplest and most successful one is primary reference fuel (PRF). PRFs are binary mixtures of $n$-heptane and iso-octane. They can represent octane number of most real gasoline fuels, except for aromatics-rich and high octane sensitivity gasoline fuels [4]. Therefore, they are widely used for studying gasoline combustion chemistry and in advanced engine research [11,12]. Chemical kinetic models are well studied for $n$ heptane and iso-octane. The first well known kinetic model for nheptane was proposed in 1998 by Curran et al. [13], and the latest update is by Zhang et al. in 2016 [14]. For iso-octane, the first kinetic model is proposed by Curran et al. in $1998[15,16]$, and the latest update is by Atef et al. in 2017 [17]. After years of updates to the kinetic models, there still remains large gap to fill in model development. For the well-studied n-heptane, many oxygenated intermediates are recently detected and measured experimentally, but they were not included in the kinetic models [18]. Moreover, many speciation profiles cannot be well captured by kinetic model predictions. These non-regular emission species, e.g. ethylene, butadiene and formaldehyde, are important in atmospheric chemistry and pollutant modelling. Ethylene could pollute soil and water, and hamper the growth of crops [19]. Butadiene is a major precursor for soot formation, which can trigger human respiratory health issue [20]. Formaldehyde is a very toxic pollutant that can trigger leukemia and cancer [21]. Other species, e.g. propene, methane and ethane would also cause different health and environmental issues.

Advancements in combustion kinetic modelling happened over the last years, however some shortages are still present for the kinetic modelling approach. First, it is time consuming to develop detailed kinetic model. Even for the most studied n-heptane kinetic model, it took over 20 years and the models are still being developed and updated. Second, emission species profiles cannot be well predicted by kinetic model. This would hamper predictions of non-regular emission of combustion engines. Finally, even the most accurate detailed kinetic models are still needed to be reduced before being applied on advanced engine design. Therefore, there is a growing need for a better and simpler approach to describe fuel behavior.

Machine learning (ML) models could be a potential answer. Large amount of experimental data can be provided to these models to train on the data and give predictions. Since all the data is from experiments, discrepancies can be largely reduced. Furthermore, ML models are 
purely mathematical, so no chemical kinetic model is required, which can save the time and effort for model development. ML models have been applied in numerous previous works on gasoline engines emissions [22], diesel engines emissions [23], atmospheric chemistry predictions [24] and pollutant predictions [25]. In this work, we developed ML models for PRFs oxidation in jet stirred reactor. A dataset of neat fuels and their mixtures, constituting of 10 neat fuels, 6 primary reference fuels (PRF) and 6 FGX surrogates tested in jet stirred reactor, is used as training dataset for ML models. Both chemical kinetic model and ML predictions are compared with experimental data. ML models show better predictions than traditional kinetic model since ML models are trained directly on experimental data and is not subjected to any discrepancies that kinetic models have. This work introduces the application of ML for data interpretation on jet stirred reactor, and implies the great potential of application of machine learning to model other combustion chemistry phenomenon.

\section{Methodology}

\section{Jet Stirred Reactor}

A combination of neat fuels and mixtures were considered for in this study, constituting of 10 neat fuels, 6 primary reference fuels (PRF) and 6 FGX surrogates. Jet stirred reactor (JSR) experiments of these fuels were performed at King Abdullah University of Science and Technology (KAUST), Saudi Arabia. Detailed description of the experimental setup can be found in previous literature [9]. A brief description is given here. Fused silica was used to make a spherical reactor (volume $76 \mathrm{~cm} 3$ ) to prevent wall reactions. Four opposing nozzles (ID $0.3 \mathrm{~mm}$ ) were used as the inlets of the reactor to achieve perfect mixing by creating jet flow stirring. The mixing performance has been checked experimentally in previous work [26]. Fuels were vaporized at $450 \mathrm{~K}$ and then diluted with nitrogen, which also functioned as a carrier gas to introduce fuel vapor into the spherical reactor through the inner capillary inlet. Diluted oxygen was introduced into the reactor through an outer quartz channel, so that oxygen and fuel would not mix until admission into the spherical reactor. A K-type thermocouple monitored reaction temperature inside the reactor. Temperature profiles inside the reactor were measured with pure nitrogen flow and showed good uniformity (gradient $<3 \mathrm{~K} / \mathrm{cm}$ ). Controlled by MKS mass flow controllers, the gas flow rates were adjusted with the reactor temperature to achieve fixed residence time inside the reactor. The gases were extracted through a quartz microprobe. Further gas reactions of the extracted gases were frozen by a pressure-drop in the sonic-throat gas sampling. Sampled gases were analyzed by Gas Chromatography. For all fuels, experimental conditions were set as following: fuel concentration $0.25 \%$, equivalence ratio 1.0 , residence time $1 \mathrm{~s}$, temperature range $750-1100$ K.

\section{Chemical kinetic modelling}

The chemical kinetic model used in this work is the latest version issued by Lawrence Livermore National Laboratory [27]. Sub-models of $n$-heptane and iso-octane are validated against ignition delay time, flame speed and speciation profiles. Simulation software used is Chemkin Pro [28]. Perfect stirred reactor model was used. Inlet composition, residence time, temperature and pressure are set in consistent with experiments. End time of simulation is set at $50 \mathrm{~s}$ to ensure converged solutions.

\section{Feature Selection}

Features for machine learning model is a numerical vector that consist of information pertaining to input of data points in the dataset. Due to the less number of fuels considering in our dataset, we can only use dense feature space, meaning the number of features in feature space should be small. An option is to use the fraction of each component of the major components as features. However, there are 10 neat species above which we need to consider other major components in face

Table 1. The fuel species dataset used in this study along with their functional groups used.

\begin{tabular}{|c|c|c|c|c|c|c|c|}
\hline & primary & secondary & tertiary & quaternary & double bond & cyclo-ring & benzene ring \\
\hline n-butane & 2 & 2 & 0 & 0 & 0 & 0 & 0 \\
\hline cyclopentane & 0 & 5 & 0 & 0 & 0 & 1 & 0 \\
\hline cyclohexane & 0 & 6 & 0 & 0 & 0 & 1 & 0 \\
\hline n-hexane & 2 & 4 & 0 & 0 & 0 & 0 & 0 \\
\hline triptane & 5 & 0 & 1 & 1 & 0 & 0 & 0 \\
\hline 1-hexene & 1 & 3 & 0 & 0 & 1 & 0 & 0 \\
\hline 2,2,4-trimethyl-2-pentene & 5 & 0 & 0 & 1 & 1 & 0 & 0 \\
\hline toluene & 1 & 0 & 0 & 0 & 0 & 0 & 1 \\
\hline NC7H16 & 2 & 5 & 0 & 0 & 0 & 0 & 0 \\
\hline IC8H16 & 5 & 1 & 1 & 1 & 0 & 0 & 0 \\
\hline PRF 50 & 3.41 & 3.12 & 0.47 & 0.47 & 0 & 0 & 0 \\
\hline PRF 64.5 & 4.02 & 2.31 & 0.67 & 0.67 & 0 & 0 & 0 \\
\hline PRF 70 & 3.82 & 2.57 & 0.61 & 0.61 & 0 & 0 & 0 \\
\hline PRF 84 & 3.86 & 2.53 & 0.62 & 0.62 & 0 & 0 & 0 \\
\hline PRF 91.5 & 3.85 & 2.53 & 0.62 & 0.62 & 0 & 0 & 0 \\
\hline PRF 95 & 3.85 & 2.53 & 0.62 & 0.62 & 0 & 0 & 0 \\
\hline FGA & 4.02 & 1.68 & 0.82 & 0.60 & 0 & 0 & 0 \\
\hline FGC & 3.69 & 1.73 & 0.64 & 0.55 & 0 & 0 & 0.05 \\
\hline FGF & 3.16 & 1.93 & 0.61 & 0.44 & 0.08 & 0 & 0.08 \\
\hline FGG & 2.45 & 1.73 & 0.37 & 0.18 & 0.08 & 0 & 0.32 \\
\hline FGI & 3.18 & 2.34 & 0.72 & 0.34 & 0.06 & 0 & 0.04 \\
\hline FGg 2 of 9 & 2.91 & 2.28 & 0.35 & 0.12 & 0 & 0 & 0.30 \\
\hline
\end{tabular}


gasoline fuels that makes feature vector sparse. A more concise and broad feature space is one consisting of functional groups. This has been used for in previous studies to predict octane number [29] and for surrogate formulations [2]. In this study, we considered seven functional groups as the feature vector for machine learning models. This include number of primary, secondary, tertiary, quaternary carbons in fuel. Additionally, number of double bonds, number of cyclo rings and number of benzene rings are used. All the fuels used in our dataset are shown in Table 1 along with the values of seven features for each of these fuels. Additionally temperature is also used as eight input which is parameterized between $750-1100 \mathrm{~K}$, depending on the experiment data for each fuel.

\section{Machine Learning Modelling}

\section{Machine learning models}

Several machine learning (ML) models are currently being used. With the recent advances in computing capacity, advancements to the existing algorithms are being done along with development of new algorithms. Our goal in this work is to train a model based on data with a known continuous output value. Therefore, this is a supervised regression problem. A widely used model for supervised problems is support vector regression (SVR) [30], which works better for smaller datasets such as in this study. SVR uses concept of kernel to create a hyper-plane to fit the data, leaving some allowable error. The SVR function can be mathematically described by equation 1 , where $\varepsilon$ and $\xi_{i}, \xi_{i}^{*}$ are positive numbers that describe the allowable error $(\varepsilon)$ and additional error above $\varepsilon$ for a data point $\mathrm{i}$ in the training set, respectively. In this work, radial basis function (RBF) kernel is used to determine $\left\langle\omega, X_{i}\right\rangle$ in equation 1 . The hyper-parameters that were tuned are regularization parameter $(C)$ and epsilon $(\varepsilon)$, shown in equation 1. Regularization parameter controls overfitting and is varied between 0.1 and 1000 in multiplication steps of 10. Epsilon is the allowable error margin in training the model and is varied between $10^{-}$ ${ }^{1}$ to $10^{-5}$ in steps of $10^{-1}$.

$$
\begin{gathered}
\text { Minimize }_{\omega, b, \xi_{i}, \xi_{i}^{*}} \frac{1}{2}\|\omega\|^{2}+C \sum_{i=1}^{n} \xi_{i}+\xi_{i}^{*} . \\
\text { such that } Y_{i}-<\omega, X_{i}>-b \leq \varepsilon+\xi_{i} \\
<\omega, X_{i}>+b-Y_{i} \leq \varepsilon+\xi_{i}^{*} \\
\xi_{i}, \xi_{i}^{*} \geq 0 \quad \forall i
\end{gathered}
$$

Another effective ML model used for supervised regression problem is random forest regression (RFR). RFR is an additive model that makes predictions by combining decisions from a sequence of base models. Each of the base model is a decision tree trained over subsample of the full training dataset [31]. The hyper-parameters that are tuned for RFR are estimators, maximum depth, minimum sample required to split an internal node and minimum samples required to be at a leaf node. Estimators is the number of trees in a model and is varied between 1 to 64 in steps of 2 . Maximum depth is varied between 1 to 5. The other two hyper-parameters are varied between 0.1 to 0.5 in steps of 0.05 . Along with the SVR and RFR, linear regression is also used for comparison in this work. More details about these three models can be found in scikit-learn documentation [32].

\section{Results and discussion}

The machine learning model development consists of a workflow in which the data is divided into training, validation and test sets. The training set is first used to train a ML model and find the error on validation set. This training is repeated for all the hyper-parameter combinations to find the optimal hyper-parameters, that gives lowest validation error. These optimal hyper-parameters are then used to train the ML model on the training and validation set combined. This model is then used to find performance on test set. This test error represents the performance of a ML model on a dataset. The dataset used in this work consists of 10 neat species and 12 mixtures, which majorly are the mixtures of those neat species. Each of the 12 mixtures are set aside as test set once and the remaining mixtures and neat species are divided into training and validation sets in random manner. This process is done for all the ML models considered in this study viz. SVR, RFR and linear regression.

Table 2: Performance of the machine learning models used in this study.

\begin{tabular}{|l|l|l|}
\hline Machine learning model & $\begin{array}{l}\text { Mean absolute } \\
\text { error }\end{array}$ & $\begin{array}{l}\text { Regression score } \\
(\boldsymbol{\%})\end{array}$ \\
\hline Support vector regression & $5.09 \mathrm{E}-05$ & 97.75 \\
\hline Random forest regression & $1.27 \mathrm{E}-04$ & 91.07 \\
\hline Linear regression & $2.69 \mathrm{E}-04$ & 68.33 \\
\hline
\end{tabular}

The averaged test error metrics for the 12 mixture datasets are shown in Table 2 for the three ML models. As can be seen from the Table 2, SVR and RFR performs significantly better over linear regression. This is justifiable since the both SVR and RFR can capture non-linear relationship between functional groups and species concentration. Also, SVR performs marginally better over RFR. Therefore, further comparisons with kinetic modelling results will be done using SVR model. All the scripts are written in python using scikit-learn libraries [32] and are made available at GitHub repository (https://github.com/kiranyalamanchi/Speciation).

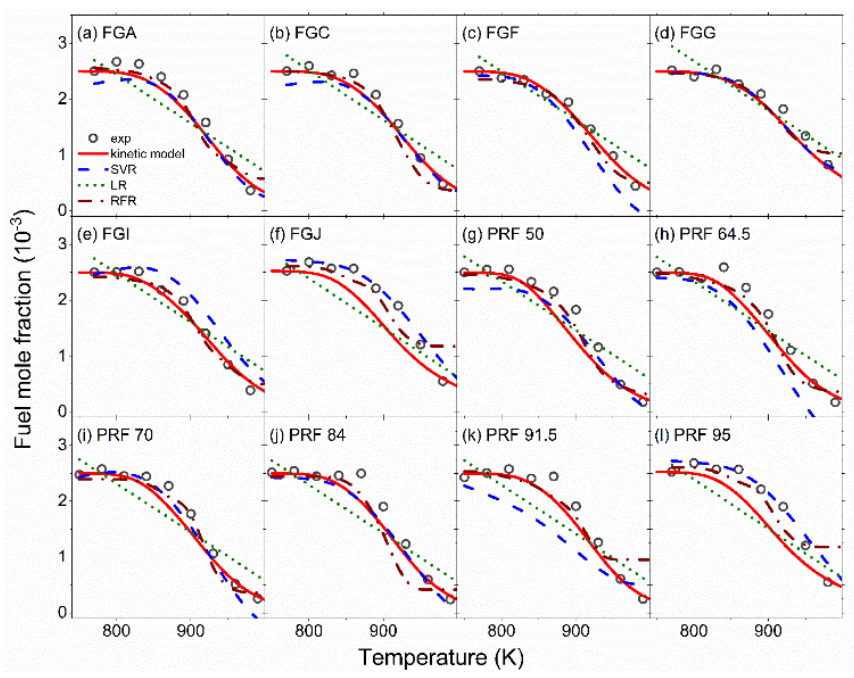

Figure 1: Comparison of fuel profiles by experiments (o), kinetic modelling ( - ) and different machine learning methods: SVR ( - - ), LR ( …...), RFR ( -. - ) . Different figures (a - i) corresponds to different fuel mixtures given in Table 1. 


\section{Comparison with kinetic modelling}

The performance of the machine learning models is compared with those of kinetic modelling as described in section 2.2. Figure 1 shows comparison for fuel profiles for the 12 fuels used in this study. Both the SVR and RFR models perform on par with the kinetic model; in few cases better than kinetic model viz. FGJ and PRF 95. It should be noted that although kinetic models are developed from the fundamental knowledge of reactions and thermodynamic data, many reaction rates are also optimized with in their uncertainty ranges. Therefore, a kinetic model can be considered as hybrid model consisting of both fundamental kinetic knowledge aswell as a regressed model over the global combustion parameters. Hence, a small discrepancy between experimental data and kinetic modelling can be observed. With machine learning models directly trained on global combustion parameters, the performance is still good without having any fundamental kinetic knowledge fed in to them.

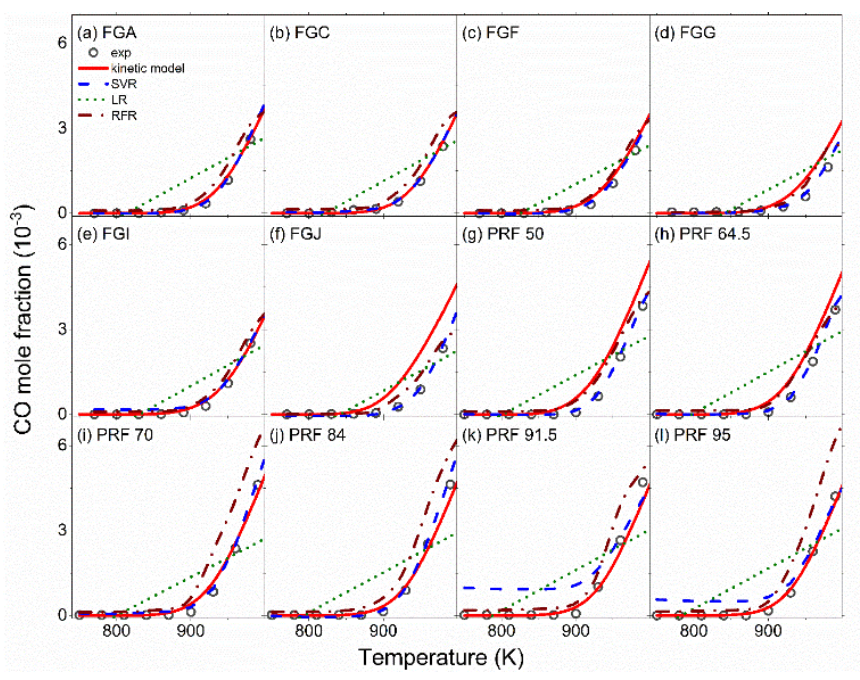

Figure 2: Comparison of $\mathrm{CO}$ profiles by experiments (o), kinetic modelling ( - ) and machine learning methods: SVR (-- ), LR ( …..), RFR ( - . - .). Different figures (a -i) corresponds to different fuel mixtures given in Table 1.

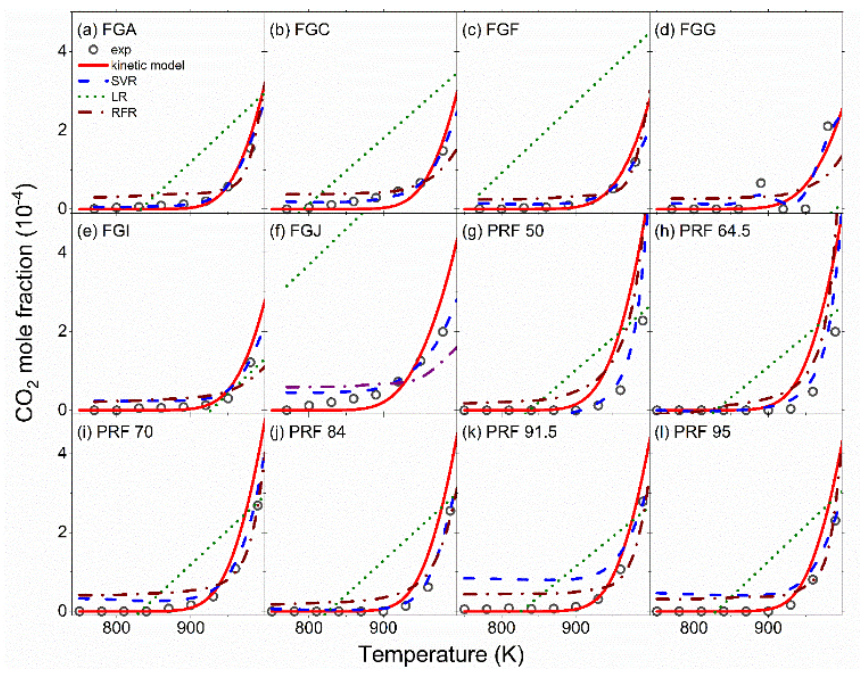

Figure 3: Comparison of $\mathrm{CO} 2$ profiles by experiments (o), kinetic modelling ( - ) and machine learning methods: SVR (-- ), LR ( ( ……, RFR ( - - - .). Different figures (a -i) corresponds to different fuel mixtures given in Table 1.
Figure 2 and 3 shows $\mathrm{CO}$ and $\mathrm{CO} 2$ profiles for all the fuel cases. The SVR and RFR models are even more closer to that of kinetic model predictions than those of fuel profiles, except for PRF 91.5 and PRF 95. In both of these cases, the machine learning models tend to predict a positive value as the saturation value for low temperatures. This could be due to the changing slopes in the experimental data although the values are closer to zero. This could however be easily avoided by taking the saturation value as zero when the slope becomes zero. Figures A1 to A4 in the appendix shows the comparison for intermediate species profiles of hydrogen, methane, ethylene and propene. For the intermediate profiles, it is observed that the data driven models predict well over the kinetic model.

\section{Conclusions}

Hydrocarbon fuels emit a lot of pollutants up on their combustion, which needs to be monitored and controlled. The traditional approach to estimate these from kinetic modelling poses limitations. In this work, an alternative method is explored taking gasoline surrogate fuels as a test case. Two Machine Learning models SVR and RFR are compared against the kinetic modelling for 12 mixture fuels. This comparison is done for fuel consumption profiles and species fraction profiles of $\mathrm{CO}, \mathrm{CO}_{2}, \mathrm{H}_{2}, \mathrm{CH}_{4}, \mathrm{C}_{2} \mathrm{H}_{4}, \mathrm{C}_{3} \mathrm{H}_{6}$. It is found that the machine learning models match the speciation profiles relative to kinetic modelling. This is therefore promising for future of modelling pollutants since there are significant advantages for data science models for speciation estimation:

1) No fundamental chemistry information is needed to develop these models.

2) It is inexpensive to both develop and use the machine learning models.

3) As the new experimental data gets generated, these models can easily incorporate the new data.

Therefore, machine-learning models can be alternatives for the pollutant speciation estimation provided there is a good database to build the models. As the new experimental data gets generated, the data science models become wider in their scope and in their potential.

\section{References}

1. Sarathy, S.M.M., Farooq, A., and Kalghatgi, G.T.G.T., "Recent progress in gasoline surrogate fuels," Prog. Energy Combust. Sci. 65:1-42, 2018, doi:10.1016/j.pecs.2017.09.004.

2. Ahmed, A., Goteng, G., Shankar, V.S.B., Al-Qurashi, K., Roberts, W.L., and Sarathy, S.M., "A computational methodology for formulating gasoline surrogate fuels with accurate physical and chemical kinetic properties," Fuel 143:290-300, 2015, doi:https://doi.org/10.1016/j.fuel.2014.11.022.

3. Javed, T., Ahmed, A., Lovisotto, L., Issayev, G., Badra, J., Sarathy, S.M., and Farooq, A., "Ignition studies of two lowoctane gasolines," Combust. Flame 185:152-159, 2017, doi:https://doi.org/10.1016/j.combustflame.2017.07.006.

4. Sarathy, S.M., Kukkadapu, G., Mehl, M., Javed, T., Ahmed, A., Naser, N., Tekawade, A., Kosiba, G., AlAbbad, M., Singh, E., Park, S., Rashidi, M. Al, Chung, S.H., Roberts, W.L., Oehlschlaeger, M.A., Sung, C.J., and Farooq, A., "Compositional effects on the ignition of FACE gasolines," Combust. Flame 169:171-193, 2016, doi:10.1016/j.combustflame.2016.04.010.

5. Sarathy, S.M., Kukkadapu, G., Mehl, M., Wang, W., Javed, T., Park, S., Oehlschlaeger, M.A., Farooq, A., Pitz, W.J.,

Page 4 of 9 
and Sung, C.-J., "Ignition of alkane-rich FACE gasoline fuels and their surrogate mixtures," Proc. Combust. Inst. 35(1):249-257, 2015,

doi:https://doi.org/10.1016/j.proci.2014.05.122.

6. Mannaa, O., Mansour, M.S., Roberts, W.L., and Chung, S.H., "Laminar burning velocities at elevated pressures for gasoline and gasoline surrogates associated with RON," Combust. Flame 162(6):2311-2321, 2015, doi:https://doi.org/10.1016/j.combustflame.2015.01.004.

7. Chen, B., Togbé, C., Selim, H., Dagaut, P., and Sarathy, S.M., "Quantities of Interest in Jet Stirred Reactor Oxidation of a High-Octane Gasoline," Energy \& Fuels 31(5):55435553, 2017, doi:10.1021/acs.energyfuels.6b03193.

8. Chen, B., Togbé, C., Wang, Z., Dagaut, P., and Sarathy, S.M.M., "Jet-stirred reactor oxidation of alkane-rich FACE gasoline fuels," Proc. Combust. Inst. 36(1):517-524, 2017, doi:10.1016/j.proci.2016.05.040.

9. Chen, B., Wang, Z., Wang, J.-Y., Wang, H., Togbé, C., Alonso, P.E.Á., Almalki, M., Mehl, M., Pitz, W.J., Wagnon, S.W., Zhang, K., Kukkadapu, G., Dagaut, P., and Mani Sarathy, S., "Exploring gasoline oxidation chemistry in jet stirred reactors," Fuel 236:1282-1292, 2019, doi:https://doi.org/10.1016/j.fuel.2018.09.055.

10. Selim, H., Mohamed, S.Y.S.Y., Dawood, A.E.A.E., and Sarathy, S.M.M., "Understanding premixed fl ame chemistry of gasoline fuels by comparing quantities of interest," Proc. Combust. Inst. 36(1):1203-1211, 2017, doi:10.1016/j.proci.2016.06.127.

11. Cai, L. and Pitsch, H., "Optimized chemical mechanism for combustion of gasoline surrogate fuels," Combust. Flame 162(5):1623-1637, 2015, doi:https://doi.org/10.1016/j.combustflame.2014.11.018.

12. An, Y., Jaasim, M., Raman, V., Hernández Pérez, F.E., Sim, J., Chang, J., Im, H.G., and Johansson, B., "Homogeneous charge compression ignition (HCCI) and partially premixed combustion (PPC) in compression ignition engine with low octane gasoline," Energy 158:181-191, 2018,

doi:https://doi.org/10.1016/j.energy.2018.06.057.

13. Curran, H.J., Gaffuri, P., Pitz, W.J., and Westbrook, C.K., "A Comprehensive Modeling Study of n-Heptane Oxidation," Combust. Flame 114(1):149-177, 1998, doi:https://doi.org/10.1016/S0010-2180(97)00282-4.

14. Zhang, K., Banyon, C., Bugler, J., Curran, H.J., Rodriguez, A., Herbinet, O., Battin-Leclerc, F., B'Chir, C., and Heufer, K.A., "An updated experimental and kinetic modeling study of n-heptane oxidation," Combust. Flame 172:116-135, 2016, doi:https://doi.org/10.1016/j.combustflame.2016.06.028.

15. Curran, H.J., Gaffuri, P., Pitz, W.J., and Westbrook, C.K., "A comprehensive modeling study of iso-octane oxidation," Combust. Flame 129(3):253-280, 2002, doi:https://doi.org/10.1016/S0010-2180(01)00373-X.

16. Curran, H.J., Pitz, W.J., Westbrook, C.K., Callahan, G. V, and Dryer, F.L., "Oxidation of automotive primary reference fuels at elevated pressures," Symp. Combust. 27(1):379-387, 1998, doi:https://doi.org/10.1016/S0082-0784(98)80426-8.

17. Atef, N., Kukkadapu, G., Mohamed, S.Y., Rashidi, M. Al, Banyon, C., Mehl, M., Heufer, K.A., Nasir, E.F., Alfazazi, A., Das, A.K., Westbrook, C.K., Pitz, W.J., Lu, T., Farooq, A., Sung, C.-J., Curran, H.J., and Sarathy, S.M., "A comprehensive iso-octane combustion model with improved thermochemistry and chemical kinetics," Combust. Flame 178:111-134, 2017,

doi:https://doi.org/10.1016/j.combustflame.2016.12.029.

18. Wang, Z., Chen, B., Moshammer, K., Popolan-Vaida, D.M.,

Page 5 of 9
Sioud, S., Shankar, V.S.B., Vuilleumier, D., Tao, T., Ruwe, L., Bräuer, E., Hansen, N., Dagaut, P., Kohse-Höinghaus, K., Raji, M.A., and Sarathy, S.M., "n-Heptane cool flame chemistry: Unraveling intermediate species measured in a stirred reactor and motored engine," Combust. Flame 187:199-216, 2018,

doi:https://doi.org/10.1016/j.combustflame.2017.09.003.

19. Ghanta, M., Fahey, D., and Subramaniam, B.,

"Environmental impacts of ethylene production from diverse feedstocks and energy sources," Appl. Petrochemical Res. 4(2):167-179, 2014, doi:10.1007/s13203-013-0029-7.

20. Catallo, W.J., Kennedy, C.H., Henk, W., Barker, S.A., Grace, S.C., and Penn, A., "Combustion products of 1,3butadiene are cytotoxic and genotoxic to human bronchial epithelial cells," Environ. Health Perspect. 109(9):965-971, 2001, doi:10.1289/ehp.01109965.

21. Heck, H. d'A and Casanova, M., "The implausibility of leukemia induction by formaldehyde: a critical review of the biological evidence on distant-site toxicity.," Regul. Toxicol. Pharmacol. 40(2):92-106, 2004,

doi:10.1016/j.yrtph.2004.05.001.

22. Sayin, C., Ertunc, H.M., Hosoz, M., Kilicaslan, I., and Canakci, M., "Performance and exhaust emissions of a gasoline engine using artificial neural network," Appl. Therm. Eng. 27(1):46-54, 2007, doi:https://doi.org/10.1016/j.applthermaleng.2006.05.016.

23. Parlak, A., Islamoglu, Y., Yasar, H., and Egrisogut, A., "Application of artificial neural network to predict specific fuel consumption and exhaust temperature for a Diesel engine," Appl. Therm. Eng. 26(8):824-828, 2006, doi:https://doi.org/10.1016/j.applthermaleng.2005.10.006.

24. Gardner, M.W. and Dorling, S.R., "Artificial neural networks (the multilayer perceptron) - a review of applications in the atmospheric sciences," Atmos. Environ. 32(14):2627-2636, 1998, doi:https://doi.org/10.1016/S13522310(97)00447-0.

25. Cai, M., Yin, Y., and Xie, M., "Prediction of hourly air pollutant concentrations near urban arterials using artificial neural network approach," Transp. Res. Part D Transp. Environ. 14(1):32-41, 2009, doi:https://doi.org/10.1016/j.trd.2008.10.004.

26. Ayass, W.W., Nasir, E.F., Farooq, A., and Sarathy, S.M., "Mixing-structure relationship in jet-stirred reactors," Chem. Eng. Res. Des. 111:461-464, 2016, doi:https://doi.org/10.1016/j.cherd.2016.05.016.

27. Mehl, M., Wagnon, S., Tsang, K., Kukkadapu, G., Pitz, W.J., Westbrook, C.K., Tsang, Y., Curran, H.J., Atef, N., Rachidi, M.A., Sarathy, M.S., and Ahmed, A., "A comprehensive detailed kinetic mechanism for the simulation of transportation fuels."

28. CHEMKIN-PRO R. 15112, Reaction Design. Inc., San Diego, CA., 2011.

29. Abdul Jameel, A.G., Oudenhoven, V. Van, Emwas, A.-H., and Sarathy, S.M., "Predicting Octane Number Using Nuclear Magnetic Resonance Spectroscopy and Artificial Neural Networks," Energy \& Fuels 32(5):6309-6329, 2018, doi:10.1021/acs.energyfuels.8b00556.

30. Drucker, H., Burges, C.J.C., Kaufman, L., Smola, A.J., and Vapnik, V., "Support Vector Regression Machines," in: Mozer, M. C., Jordan, M. I., and Petsche, T., eds., Advances in Neural Information Processing Systems 9, MIT Press: 155-161, 1997.

31. Breiman, L., "Random Forests," Mach. Learn. 45(1):5-32, 2001, doi:10.1023/A:1010933404324.

32. Pedregosa, F., Varoquaux, G., Gramfort, A., Michel, V., 
Thirion, B., Grisel, O., Blondel, M., Prettenhofer, P., Weiss, R., Dubourg, V., Vanderplas, J., Passos, A., Cournapeau, D., Brucher, M., Perrot, M., and Duchesnay, E., "Scikit-learn: Machine Learning in $\{\mathrm{P}\}$ ython," J. Mach. Learn. Res. 12:2825-2830, 2011.

\section{Contact Information}

Kiran K. Yalamanchi, Kiran.yalamanchi@kaust.edu.sa; Binjie Chen, bingjie.chen@kaust.edu.sa; S. Mani Sarathy, mani.sarathy@kaust.edu.sa; Clean Combustion Research Center, King Abdullah University of Science and Technology (KAUST)

\section{Acknowledgements}

This work was supported by King Abdullah University of Science and Technology (KAUST) Office of Sponsored Research under the award number OSR-2019-CRG7-4077, and the KAUST Clean Fuels Consortium (KCFC) and its member companies. 
Page 7 of 9

7/20/2015 


\section{Appendix}

\section{Intermediate profiles:}

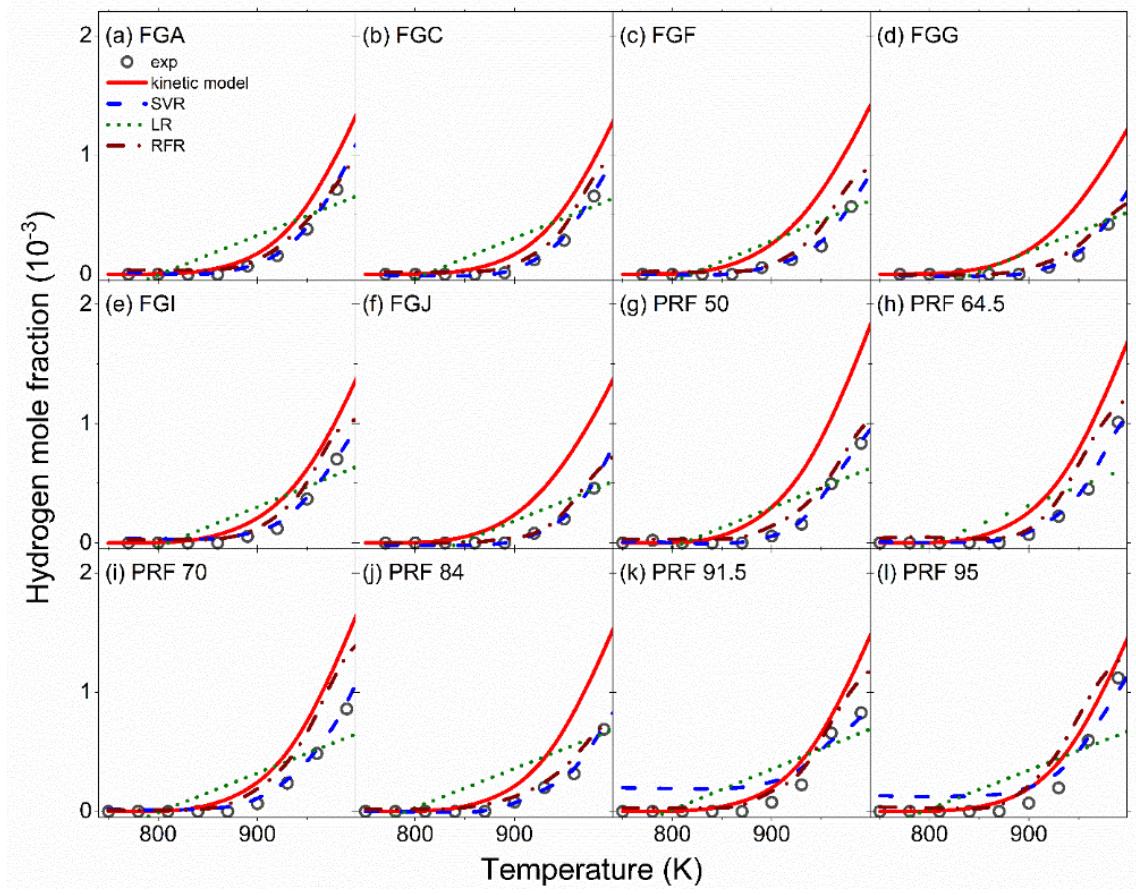

Figure A1: Comparison of hydrogen profiles by experiments, kinetic modelling and different machine learning methods.

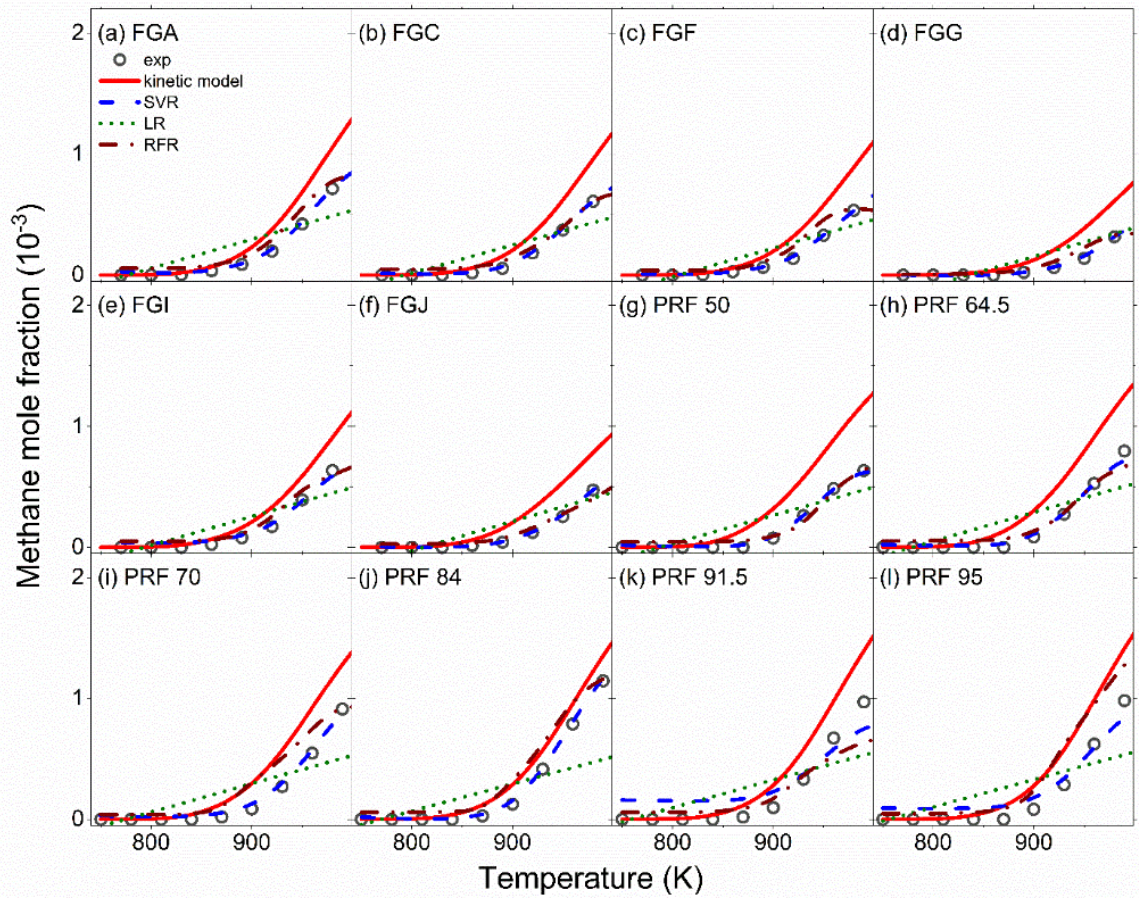

Figure A2: Comparison of methane profiles by experiments, kinetic modelling and different machine learning methods. 


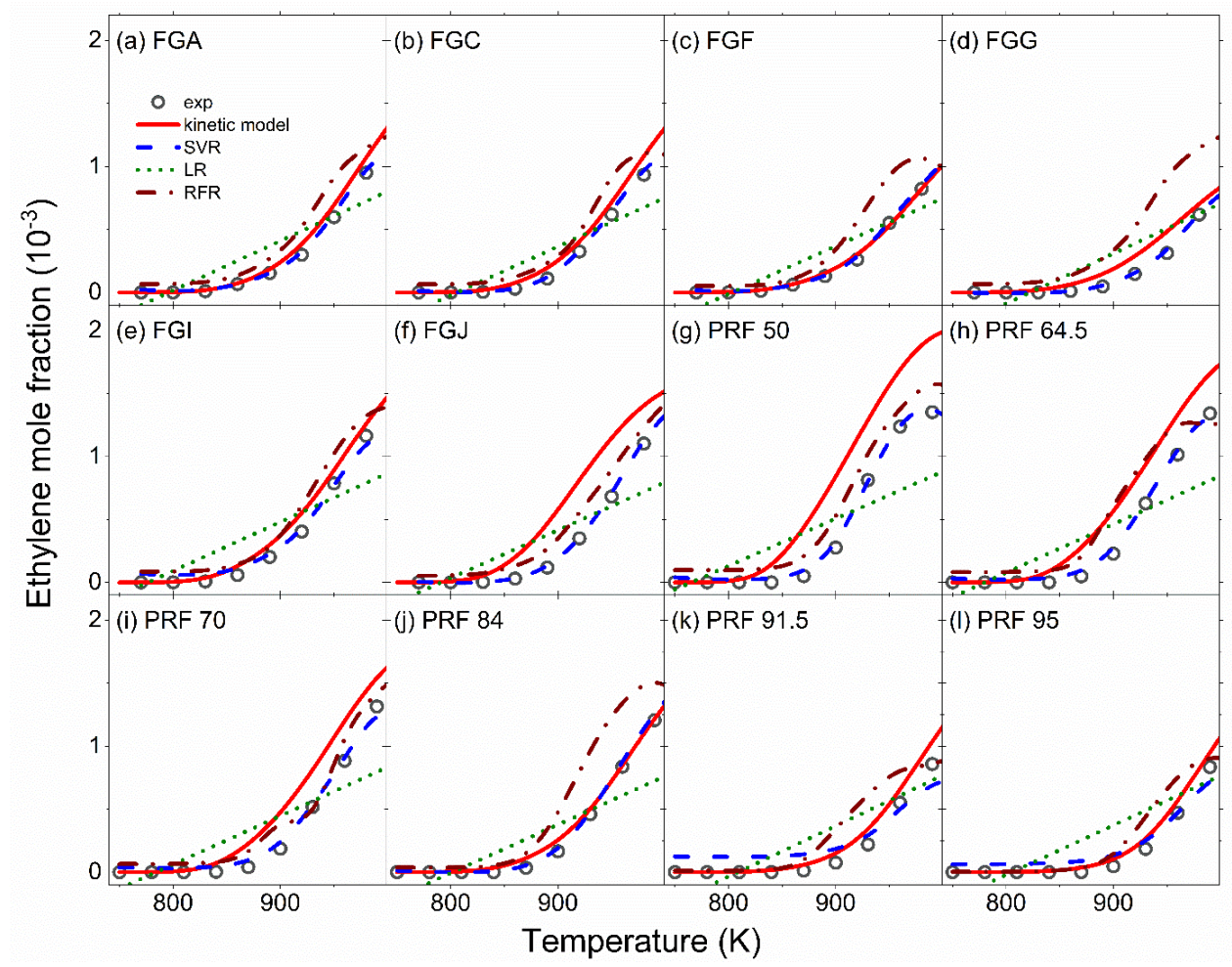

Figure A3: Comparison of ethylene profiles by experiments, kinetic modelling and different machine learning methods.

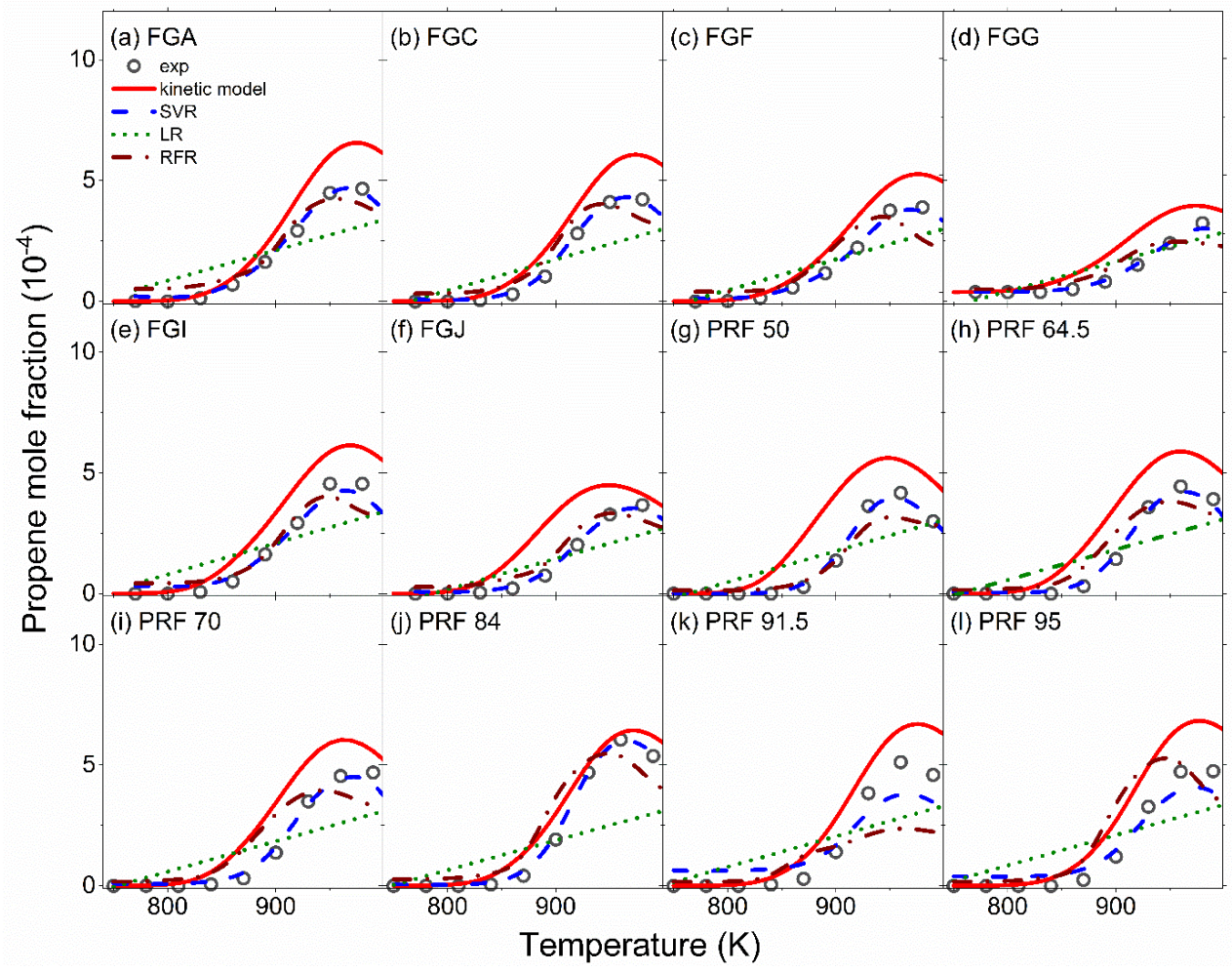

Figure A4: Comparison of propene profiles by experiments, kinetic modelling and different machine learning methods. 